\title{
Surface melting of copper by ultrashort laser pulses
}

\author{
J. Vincenc Oboňa ${ }^{1}$, V. Ocelík ${ }^{1}$, J. Th. M. De Hosson ${ }^{1}$, \\ J. Z. P. Skolski ${ }^{2,3}$, V. S. Mitko ${ }^{2,3}$, G. R. B. E. Römer ${ }^{3}$ \\ \& A. J. Huis in 't Veld ${ }^{3,4}$ \\ ${ }^{I}$ Materials Innovation Institute M2i, Department of Applied Physics, \\ University of Groningen, The Netherlands \\ ${ }^{2}$ Materials Innovation Institute M2i, The Netherlands \\ ${ }^{3}$ University of Twente, Faculty of Engineering Technology, \\ The Netherlands \\ ${ }^{4}$ TNO Science \& Industry, Department Materials Technology, \\ The Netherlands
}

\begin{abstract}
The main advantage of ultrashort laser pulses in manufacturing technology is their very high removal rate of material and high quality of microstructures with the smallest dimensions at $1 \mu \mathrm{m}$ level. The accuracy is mainly due to an almost absence of thermal diffusion into bulk material. In this paper we report the investigation on polycrystalline $\mathrm{Cu}$ sample surface treated by 6.7 ps laser pulses with $1030 \mathrm{~nm}$ laser light wavelength. Scanning electron microscopy micrographs reveal the presence of jet-like structures with spherical drop-like endings, solidified spheres and many bubble bursts at even lower fluence than the threshold value for the ablation is. Within the molten material the jet-like features are due to an explosion of bubbles originated in solid-liquid-vapor transitions. In the case of below-threshold irradiation, the same objects can be seen along surface scratches, dot contaminations etc., which indicate an increase of the laser light absorption on these inhomogeneities.

Keywords: electron microscopy, laser pulses, copper, bubbles, jets, membrane.
\end{abstract}




\section{Introduction}

The modification of a metal surface by ultrashort pulsed laser light is under intense investigation because of fundamental knowledge of laser-matter interaction and its potential use in industry [1-3]. The main advantage is the almost negligible thermal diffusion in the material. Process of laser light absorption and thermodynamics of the absorbed energy relaxation determines the appearance of a metal surface after the laser treatment. Two the most important parameters influencing the irradiated material temperature profile are the electron thermal conductivity (ETC) and the electron-phonon coupling (EPC) strength. These parameters are strongly material dependent and apply on the fluence as well $[4,5]$. In general it could be stated that for fluences higher than ablation threshold the treated material undergoes solid-gas or solid-liquid-gas transitions according to the material temperature at a particular depth in the irradiated volume.

In the present article, the treatment of polished polycrystalline $\mathrm{Cu}$ samples by 6.7 ps linearly polarized laser pulses with $1030 \mathrm{~nm}$ wavelength is reported. Investigation of the samples by Scanning Electron Microscopy (SEM) shows the signs of the material melting in the form of solidified exploding bubbles, jets with spherical endings and thin membranes at fluences slightly larger than ablation threshold. According to our knowledge, observations of the bubbles bursts have not been reported before.

\section{Experimental}

An Ytterbium-doped YAG (Yb: YAG) system (Triumph TruMicro) with a central wavelength of $1030 \mathrm{~nm}$ was used for machining of $99.995 \%$ purity copper samples. The laser system generates laser pulses (repetition rate $50 \mathrm{kHz}$ ) with a maximum average power of $50 \mathrm{~W}$. We used $0.3-5 \mathrm{~W}$ power for the samples treatment. The duration of Gaussian shaped laser pulse was 6.7 ps in all experiments. A combination of a rotary $\lambda / 2$ wave plate and a beam splitting cube served as a power attenuator. The laser light was linearly polarized. Manipulation of the beam on the sample was accomplished by moving of the sample stage. A $100 \mathrm{~mm}$ telecentric f-theta lens (Ronar of Linos, Germany) for $1030 \mathrm{~nm}$ wavelength focused the beam to a circular spot with diameter of $26 \mu \mathrm{m}$. The average power was measured at the exit of the scanner system by a power meter. In all experiments the normal incidence of laser light was used.

$\mathrm{Cu}$ samples were polished in several polishing steps to final root-mean-square (RMS) roughness lower than $10 \mathrm{~nm}$. A preparation procedure recommended by Struers Co. was employed [6]. This procedure consists of mechanical grinding with SiC-paper number 220 and $9 \mu \mathrm{m}$ diamond suspension, polishing with $3 \mu \mathrm{m}$ diamond suspensions and final polishing with OPU suspension of $40 \mathrm{~nm} \mathrm{SiO}_{2}$ particles.

Scanning Electron Microscopy technique was used to investigate the sample surfaces treated by ultrashort laser pulses. A Philips XL30 SEM equipped with a field emission gun offers a lateral resolution of the surface objects at a level of 
few nanometers. The lack of height information of the objects was partially compensated by observations on tilted surfaces. 3D maps of laser spots as well as RMS roughness of pre polished surface were obtained by use of confocal Microscopy ( $\mu$ Surf Nanofocus optical confocal microscope).

\section{Results}

\section{SEM observations}

On the highly polished $\mathrm{Cu}$ surfaces we performed experiments in order to find threshold laser fluence for the material ablation and description of surface features created with such an intensive laser light impingement. These experiments were performed by use of Scanning Electron Microscopy.

Spatial fluence profile $\Phi(\mathrm{r})$ for laser beam with Gaussian distribution of energy is defined as

$$
\Phi(r)=\Phi_{0} \exp \left[-\frac{2 r^{2}}{\omega_{0}^{2}}\right],
$$

where $r$ is distance from the beam centre, $\omega_{0}$ is beam radius at $1 / \mathrm{e}^{2}$ value of maximum laser beam fluence $\Phi_{0}$. The maximum laser fluence is related to laser pulse energy $\mathrm{E}_{\mathrm{P}}$ as follows

$$
\Phi_{0}=\frac{2 E_{P}}{\pi \omega_{0}^{2}} .
$$

Finally, the combination of these two equations and substitution of radius $r$ by diameter $\mathrm{D}[7,8]$ leads to

$$
D^{2}=2 \omega_{0}^{2} \ln \left(\frac{\Phi_{0}}{\Phi_{t h}}\right) .
$$

Using the eqn. (3), it is easy to find the threshold value of laser beam fluence $\Phi_{\text {th }}$ for all surface events appearing on the sample surface in circular concentric arrangement with a diameter $D$. Moreover, the slope $2 \omega_{0}{ }^{2}$ of $D^{2}=f\left(\ln \left(E_{P}\right)\right)$ function gives us the exact value of $1 / \mathrm{e}^{2}$ beam diameter without difficulties related to use of a beam-profiler equipment for determining it.

Initial RMS roughness of less than $10 \mathrm{~nm}$ level is significantly augmented by the ultrashort laser pulse impingement. In the Figure 1 the evident signs of the material melting and phase explosions are present in the form of exploding bubbles, jets with spherical endings as well as thin membranes which surround scratches on the sample surface.

The detail inspection of the Figure 1 reveals the solidification of exploding bubbles in different stages. Close-up of the picture shows one bubble just after the burst. A thicker material rim encloses incipient hole in the thin bubble membrane. The similar bubble bursts are also present within deeper scratches. These bursts are clearly visible in the scratches at distances even much larger 
than diameter of circle where, at smooth surface, the fluence exceeds ablation threshold. In the scratch 1 , an increase of bubble diameter clearly corresponds to its decreasing distance from the beam centre. In the middle of scratch 2 the small ripple-like structures orthogonal to the scratch side wall are well visible. In the case of side view at the scratch 3 one can see material ejected from the scratch in the form of thin membranes.

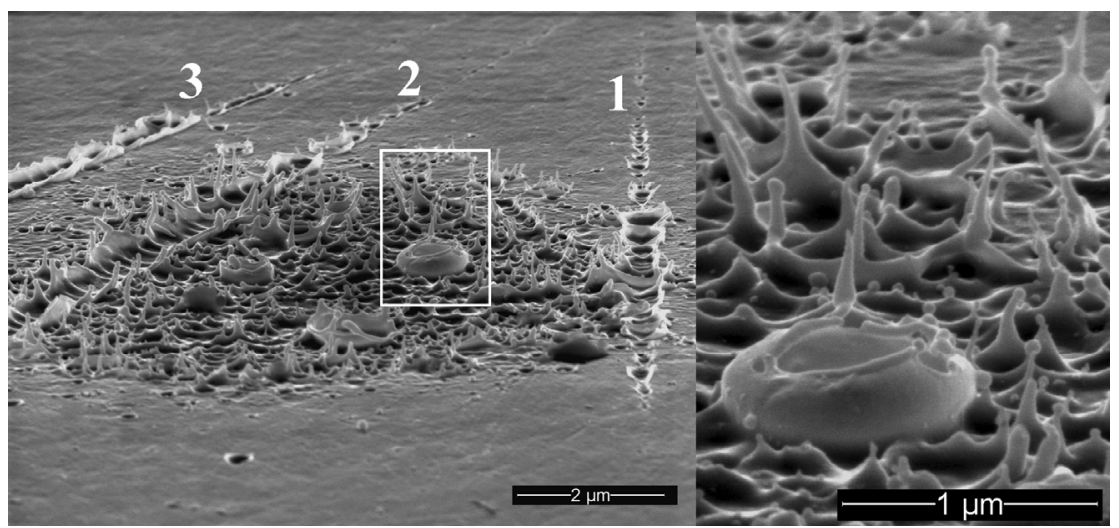

Figure 1: $\quad \mathrm{Cu}$ sample surface threaded by $6.7 \mathrm{ps}$ single laser pulse with energy $10 \mu \mathrm{J}$. Close-up of the laser pulse central part depicts bubble burst and many jet features with drop-like spherical endings. The sample is tilted vertically $75^{\circ}$ from the normal view. Numbers 1,2 and 3 mark the random scratches.

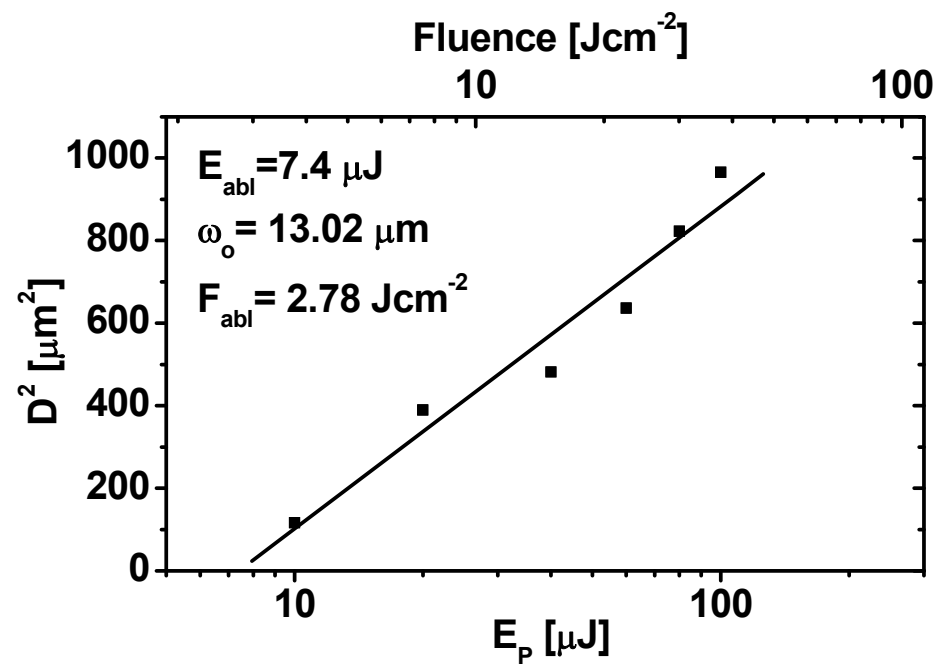

Figure 2: $\quad$ Graph of squared laser spot diameter dependence on pulse energy and pulse peak fluence. 
Diameters of the ablated areas were measured with SEM for pulse energies in the range from $6 \mu \mathrm{J}$ to $100 \mu \mathrm{J}$. Squared laser spot diameter dependence on the logarithm of pulse energy was drawn (Figure 2) in order to find out the ablation pulse energy, the $1 / \mathrm{e}^{2}$ laser beam radius and the ablation threshold fluence. These are $7.40 \mu \mathrm{J}, 13.02 \mu \mathrm{m}$ and $2.78 \mathrm{Jcm}^{-2}$, respectively.

The same copper sample treated by $50 \mu \mathrm{J}$ laser pulse energy (Figure 3 ) shows the absence of solidified exploding bubbles in the centre of the spot. At the energy 5 times higher in comparison to the previous picture vertical jets with the spherical endings are present in the centre of the laser spot.

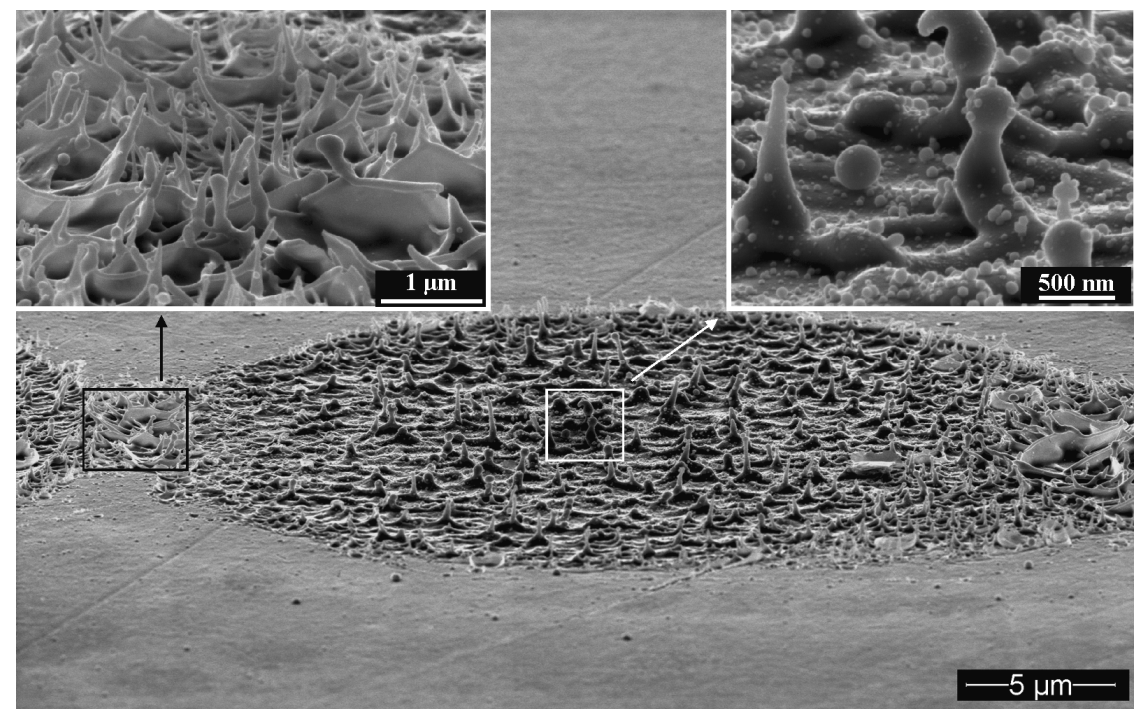

Figure 3: $\quad \mathrm{Cu}$ sample surface threaded by $6.7 \mathrm{ps}$ single laser pulse with energy $50 \mu \mathrm{J}$. The sample is tilted vertically $75^{\circ}$ from the normal view. Close-ups show details of features in the laser spot center and slight overlap of two adjacent pulses, respectively.

Close-up of central part of the laser spot reveals a surface covered by many solidified spheres with widely distributed diameters with their maximum at $\sim 250 \mathrm{~nm}$. In the case of slightly overlapping laser pulses the sample surface is modified in a different way. Melting of the surface is represented by jets ejections and a creation of thin membranes is significant. Thickness of the membranes could be estimated as 100-200 nm from the semi-transparency of the membranes for electrons.

\section{Discussion}

Modification of a metal surface by ultrashort laser pulses strongly depends on processes of laser light absorption and relaxation in the material. The absorption is governed by Lambert-Beer law and in a metal it occurs exclusively via inverse 
process to Bremsstrahlung. The absorption generates a highly non-equilibrium states of excited electrons. These non-equilibrium electrons are thermalized via electron-electron scattering within few hundreds of femtoseconds [9]. After the thermalization, equilibrium electron temperature $T_{e}$ and Fermi distribution are established. From this moment on, the diffusion of hot electrons is driven by the thermal gradient but there is still a minor amount of non-equilibrium electrons penetrating deeply under the surface due to ballistic transport [10]. Next step of the absorbed energy transfer is electron-phonon coupling which occurs in the range of $\sim 1-50 \mathrm{ps}$ (depending on a metal [11]) after front part of the laser pulse impinges the surface. As mentioned in the introduction, two competing processes, electron thermal conductivity (ETC) and electron-phonon coupling (EPC), determine how large the spread of the absorbed energy is in the material and how high the surface temperatures could be expected for a certain metal. However, determining of the surface temperature is complicated because of electron gas temperature dependence of the parameters (especially for noble metals). Taking into account all mentioned aspects of laser-matter interaction one could expect a creation of intricate temperature profile of the material lattice after electron-phonon energy transfer [12]. It means for materials with high surface lattice temperature, the ablation processes called vaporization and fragmentation may be dominant. On the other hand for lower surface temperatures or deeper parts of the irradiated volume the explosive boiling, simple melting and photochemical process (spallation) are more probable.

Our experiments show a significant amount of solidified liquid features on the $\mathrm{Cu}$ samples (Figure 1). The existence of solidified bubbles in different stages after the burst is a strong suggestion of phase explosions in the irradiated material. Moreover, a presence of their different stages indicates that there are plenty of the competing explosions within the irradiated area in the time range from start of the ablation until the surface is completely frozen. Two interesting details remain: the holes on the bubbles surrounded with a thicker rim and the spherical endings of the jets are very similar to those on bursting water bubbles [13]. Origin of the thicker rim is in equilibration of inner bubble and ambient pressure. During the process, strong vapor flow through the holes opening folds the film border outwards. Creation of the jets with spherical ends originates in competition of inertial, viscous and capillary forces [13]. Under certain conditions the rim of the retracting film on the expanding bubble may collapse into tendrils and droplets. At higher energy applied (Figure 3), only vertically standing jets with the spherical endings are present in the laser spot and plenty of solidified spheres are lying on and around it. The change of the picture to the Figure 1 could be explained by different hydrodynamic (higher material temperature $=$ lower surface tension and viscosity for $\mathrm{Cu}$ ) and inertial (faster expansion) conditions at the $5 \mathrm{x}$ higher applied fluence. For the condition of higher inertial forces and simultaneous acting of surface tension, larger amount of liquid droplets may be extracted from the material tendrils. This technique seems to be capable for the production of metal sub-micron spheres. However, from the industrial point of view, it is hard to produce the spheres with 
homogeneous diameter due to Gaussian distribution of the radiation and material nonhomogeneities as well.

Slightly overlapping areas of the adjacent laser spots are covered with many thin material membranes and jets, much more pronounced than on the border of the ablated area. The difference in laser-matter interaction on already modified surface with the previous pulse could be explained by completely different surface properties (roughness, absorbance, reflectance, refractive index etc.).

In the Figure 1 the explosions of bubbles are found within scratches even for fluences lower than ablation threshold at smooth surface in their surrounding. In this case the temperature sufficient for the melting and phase explosions is reached via multiple reflections on side walls of the scratches, i.e. larger portion of absorbed fluence. The same is valid for dot non-homogeneities as well.

As seen in the scratch 2 in the Figure 1, the bottom of the scratch is covered by ripple-like structures with a direction orthogonal to side walls of the scratch. Creation of these structures we explain as solidified common walls of the adjacent exploding bubbles and it can be applied for explanation of High Frequency Surface Laser Induced Periodic Surface Structures between Low Frequency Surface Laser Induced Periodic Surface Structures on other metals [14].

Ablation threshold fluence of $2.78 \mathrm{Jcm}^{-2}$ has been found experimentally. It has a higher value in comparison to $1.7 \mathrm{Jcm}^{-2}$ reported in [15]. Problem of the dispersion of experimental values of ablation threshold could lie in poor comparability of the experimental set-ups. For instance, reflectivity of copper surface is around $95 \%$ in near IR [15]. In the case of only 5\% laser light absorption, value of initial surface roughness could influence the absorption significantly (see scratches in Figure 1). The second reason of our higher value could be caused by use of longer laser pulses (6.7 ps) in comparison to $100 \mathrm{fs}$ in [15]. Work [5] already suggested a complicated dependence of maximal surface temperature on the pulse duration for $\mathrm{Cu}$.

\section{Conclusions}

In this work the ultrashort laser pulse treatment of highly polished $\mathrm{Cu}$ samples is studied. Surface modifications induced by 6.7 ps linearly polarized laser pulses with $1030 \mathrm{~nm}$ wavelength were investigated by Scanning Electron Microscopy. The results are concluded as follows:

- Ablation pulse energy, $1 / \mathrm{e}^{2}$ laser beam radius and ablation threshold fluence are $7.40 \mu \mathrm{J}, 13.02 \mu \mathrm{m}$ and $2.78 \mathrm{Jcm}^{-2}$, respectively.

- Surface at slightly over-threshold value of fluence revealed many solidified bubbles explosion and jets with drop-like ends.

- The same structures can be obtained even for lower fluences than ablation threshold if the low energy pulse impinges scratches or material inhomogeneities on the sample surface.

- At higher fluences the presence of exploding bubbles is only at a margin of the ablated area where the fluence is close to ablation threshold. The 
central area is covered only with vertically arranged jets with spherical endings without any presence of exploding bubbles.

- If the laser pulse impinges the sample surface already modified with the previous pulses, the surface gets much higher portion of jets with spherical endings and parts of thin membranes.

- The surface of laser spot and its close surrounding are covered at higher fluences by solidified spheres with wide distribution of diameters with a maximum at $\sim 250 \mathrm{~nm}$.

Predictions of the material lattice temperature in a particular depth just after electron-phonon temperature equalization is difficult. In the laser-mater interaction many electron temperature dependent parameters are involved. A lot of computation and experimental work have to be done for detail understanding of the ultrashort laser-matter interaction.

\section{Acknowledgement}

This research was carried out under project number M61.3.08300 in the framework of the Research Program of the Materials innovation institute M2i (www.m2i.nl).

\section{References}

[1] Huis in 't Veld, B., Groenendijk, M., Fischer, H., On the Origin, Growth and Application of Ripples, JLMN-Journal of Laser Micro/ Nanoengineering, 3, pp. 206-210, 2008.

[2] Vorobyev, A.Y., Guo, C.L., Femtosecond laser nanostructuring of metals, OPTICS EXPRESS, 14, pp. 2164-2169, 2006.

[3] Weck, A., Crawford, T.H.R., Wilkinson, D.S., Haugen, H.K., Preston, J.S., Laser drilling of high aspect ratio holes in copper with femtosecond, picosecond and nanosecond pulses, Appl. Phys. A, 90, pp. 537-543, 2008.

[4] Lin, Z., Zhigilei, L. V., Celli, V., Electron-phonon coupling and electron heat capacity of metals under conditions of strong electron-phonon nonequilibrium, Phys. Rev. B, 77, 075133(1)-(17), 2008.

[5] Ivanov, D. S., Rethfeld, B., The effect of pulse duration on the interplay of electron heat conduction and electron-phonon interaction: Photo-induced versus photo-thermal damage of metal targets, Appl. Surf. Sci., 255, 97249728, 2009.

[6] http://www.struers.com

[7] Bonse, J., Wrobel, J.M., Krüger, J. \& Kautek, W., Ultrashort-pulse laser ablation of indium phosphite in air, Appl. Phys. A, 72, pp. 89-94, 2001.

[8] Ben-Yakar, A. \& Byer, R.L., Femtosecond laser ablation properties of borosilicate glass, J. Appl. Phys., 96(9), pp. 5316-5323, 2004.

[9] Retfeld, B., Kaiser, A., Vicanek, M., Simon, G., Femtosecond laser-induced heating of electron gas in aluminum, Appl. Surf. Sc., 96, pp. 109-112, 1996. 
[10] Hohlfeld, J., Wellershoff, S.-S., Gudde, J., Conrad, U., Jahnke, V., Matthias, E., Electron and lattice dynamics following optical excitation of metals, Chemical Physics, 251, 237-258, 2000.

[11] Breitling, D., Ruf, A., Dausinger, F., Photon Processing in Microelectronics and Photonics III, Society of Photo-optical Instrumentation Engineers (SPIE), pp. 49-63, 2004.

[12] Lewis L. J., Perez D., Laser ablation with short and ultrashort laser pulses: Basic mechanism from molecular-dynamics simulations, Appl. Surf. Sci., 255, 5101-5106, 2009.

[13] Bird, J.C., de Ruiter, R., Courbin, L., Stone, H.A., Daughter bubble cascades produced by folding of ruptured thin films, Nature, 465, pp. 759$762,2010$.

[14] Vincenc Obona, J., Ocelík, V., Skolski, J.Z.P., Mitko, V.S., Römer, G.R.B.E., Huis in 't Veld, A.J., De Hosson, J.Th.M., On the surface topography of ultrashort laser pulse treated steel surfaces, submitted.

[15] Byskov-Nielsen, J., Savolainen, J.-M., Christensen, M.S., Balling, P., Ultrashort pulse laser ablation of metals: threshold fluence, incubation coefficient and ablation rates, Appl. Phys. A, 101, pp. 97-101, 2010. 